\section{HYDRODYNAMIC PERFORMANCE OF HEART VALVE PROSTHESES: OPEN DISCUSSION ON EUROPEAN COMMITTEE FOR STANDARDIZATION INTERNATIONAL ORGANIZATION FOR STANDARDIZATION STANDARD 5840 \\ To the Editor:}

In response to the publication of the European Committee for Standardization International Organization for Standardization (EN ISO) standard 5840 on May $2009,{ }^{1}$ which replaces the previous release of March 2006, we would like to open a discussion on the criteria used to assess the performance of cardiac valve prostheses. This EN ISO standard gives the minimum performance requirements that have to be matched by heart valve substitutes in terms of "effective orifice area $\mathrm{A}_{\mathrm{EO}}$ " for a number of given valve sizes for both aortic and mitral positions (see Table 2 in EN ISO standard 5840 ${ }^{1}$ ).

$\mathrm{A}_{\mathrm{EO}}$ depends on the pressure drop through the valve. The following formula (which derives from the Bernoulli equation) has been adopted in the EN ISO standard to calculate $\mathrm{A}_{\mathrm{EO}}{ }^{1}$ :

$$
A_{E O}=\frac{q_{V R M S}}{51.6 \times \sqrt{\frac{\Delta p}{\rho}}}
$$

where $q_{V R M S}$ is the root mean square forward flow (in milliliters per second), $\Delta p$ is the mean pressure difference measured over the positive pressure period of the forward flow phase (in millimeters of mercury), and $\rho$ is the density of the test fluid (in grams per cubic centimeter). The constant (51.6) is not dimensional; thus the equation is only valid with the specified units.

First, we point out that for pulsatileflow tests, the norm details neither the geometry of the pulse duplicator in the proximity of pressure measuring sites nor the location of these sites with respect to the midplane of the heart valve substitute sewing ring. Indeed, the ge-

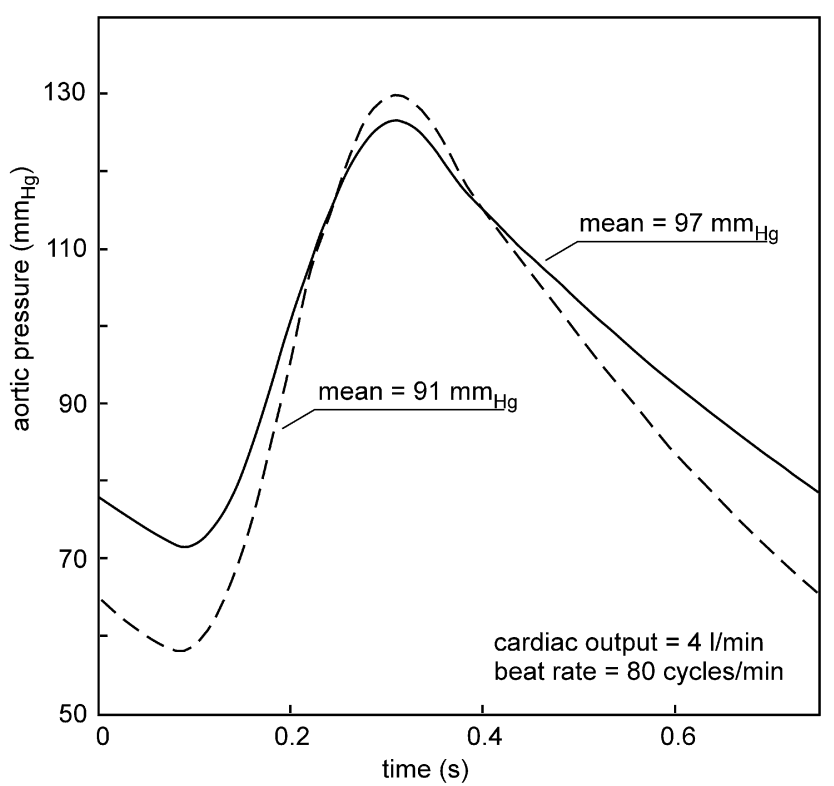

FIGURE 1. Measured aortic pressure waveforms for two biologic prosthetic valves in aortic position. Waveforms differ considerably despite comparable mean values.

ometry of the device in the vicinity of the valve housing affects the geometry of the jet through the open valve. Moreover, according to conservation of mass, jet velocity varies along flow axis as a function of jet cross-sectional area. ${ }^{2}$ According to the Bernoulli equation, the pressure (that is $\Delta \mathrm{p}$ itself) thus varies along the jet. ${ }^{2}$

Furthermore, the norm states, "The minimum performance requirements correspond to the following pulsatileflow conditions: ....mean aortic pressure $=100 \mathrm{~mm} \mathrm{Hg} \ldots . . " 1$ Pulsatile flow conditions are usually reproduced in a pulse duplicator, as reported for previous work from our laboratory ${ }^{3,4}$ in which the Sheffield pulse duplicator was used. We found that different prostheses tested in the Sheffield pulse duplicator may present comparable values of mean aortic pressure but quite different aortic pressure waveforms (data not published; Figure 1). We therefore wonder whether the mean value alone can be considered a reliable parameter for assessing prosthesis performance.

From a clinical viewpoint, any uncertainty in the assessment of prosthetic heart valve devices due to a lack of confidence in pressure measurement could result in an ambiguous characterization of their hemodynamic performance. Actually, the norm cannot prevent misestimation of the $\mathrm{A}_{\mathrm{EO}}$. As a consequence, Doppler evaluation of patients after valve replacement could estimate values of $A_{E O}$ lower than expected (or, equivalently, pressure gradients higher than expected). Misleading quantification of the degree of obstruction to flow caused by the prosthetic device is thus possible.

For these reasons, we suggest an open discussion to improve the assessment of cardiac valve prostheses.

Francesca Maria Susin, $P h D^{a}$ Andrea Bagno, $P h D^{b}$

Gino Gerosa, $M D^{c}$

${ }^{a}$ Department of Hydraulic, Maritime,

Environmental, and Geotechnical Engineering

${ }^{b}$ Department of Chemical Process Engineering

${ }^{c}$ Department of Cardiovascular Surgery, University of Padua Medical School University of Padua Padua, Italy

\section{References}

1. Cardiovascular implants—cardiac valve prostheses. EN ISO 5840:2009. ICS: 11.040.40. 2009. Available from: http://webstore.uni.com/unistore/public/product details?productId=UNINI584000-2009!EEN. 
2. Yoganathan AP, Travis BR. Fluid dynamics of prosthetic valves. In: Otto CM, ed. Practice of clinical echocardiography. 3rd ed. Philadelphia: WB Saunders; 2007:552-76

3. Bottio T, Caprili L, Casarotto D, Gerosa G. Small aortic annulus: the hydrodynamic performances of 5 commercially available bileaflet mechanical valves. J Thorac Cardiovasc Surg. 2004;128:457-62.

4. Gerosa G, Tarzia V, Rizzoli G, Bottio T. Small aortic annulus: the hydrodynamic performances of 5 commercially available tissue valves. $J$ Thorac Cardiovasc Surg. 2006;131:1058-64.

doi:10.1016/j.jtcvs.2010.01.025

BONANNO'S CATHETER: A LESS INVASIVE AND COSTEFFECTIVE

\section{ALTERNATIVE FOR DRAINAGE OF PLEURAL EFFUSION \\ To the Editor:}

It was with great interest that I read a response letter by Asopa and Patel ${ }^{1}$ to an article by Chetty and colleagues. ${ }^{2}$ Their article describes the safe use of a Bonanno catheter for drainage of a pleural effusion. Asopa and Patel called for modifications to the catheter before it was considered for general use after an incident at their center in which the left ventricle was inadvertently pierced during catheter insertion.

Traditionally, the Seldinger technique has been used to insert a pigtail drain into the intercostal space to allow the safe drainage of a pleural effusion. ${ }^{3}$ Many risks and technical difficulties come with the use of this technique. Often the needle is misplaced when the aspiration syringe is being removed, leading to drain insertion outside the chest cavity or indeed the needle being advanced into the heart or large vessels. ${ }^{4}$

The use of the Bonanno catheter as described by Chetty and associates ${ }^{2}$ is an innovative use of a suprapubic catheter and, in my experience, a simple and safe way to insert a chest drain. The technique is fully described in their article, and there are several steps that ensure that the procedure is carried out as safely as possible and the complication risk is minimized. Asopa and Patel $^{1}$ believed that the length of the catheter and the lack of external markings caused the method to become unsafe compared with the Seldinger technique. I disagree. If the method of insertion laid out clearly and concisely in the article by Chetty and associates is followed carefully, complications can be avoided. It is recommended that during local anesthetic infiltration, the parietal pleura be breached and a test aspiration carried out. This allows the operator to feel and see how far the catheter will need to be advanced; the test will ensure that the effusion is present at that intercostal level and that it is large enough to be drained safely. If there are any problems with this test aspiration, the procedure should be abandoned. Second, when advancing the catheter, one should be aspirating all the time so that as soon as the pleura is breached and the effusion is aspirated, it is apparent that the catheter is in the cavity and the operator can stop advancing the needle. The technique is then extremely simple; the catheter is advanced while the needle is being held still, similar to the technique for venous cannulation, until the catheter has been fully inserted.

I see no reason why one should lose track of how far the needle has been advanced. If the operator is able to aspirate fluid with a local anesthetic needle, then the distance the catheter and tracer need to be advanced will be relatively small. If the operator is aspirating the entire time, then the equipment should not be advanced beyond the effusion and into the heart or large vessels.

I appreciate the concerns that Asopa and Patel have expressed, and indeed markings on the catheter may prove to be helpful to some, but if Chetty and colleagues' comprehensive guidance is followed, no problems should be encountered.

Annabel Sharkey, MBChB Northern General Hospital Cardiothoracic Surgery, Sheffield South Yorkshire, United Kingdom

\section{References}

1. Asopa S, Patel A. Bonnano's catheter: A less invasive and cost-effective alternative for drainage of pleural effusion. J Thorac Cardiovasc Surg. 2006; 132:1503-4

2. Chetty GK, Elahi MM, Siddagangaiah V Leverment JN. Bonanno's catheter: a less invasive and cost-effective alternative for drainage of pleural effusion. J Thorac Cardiovasc Surg. 2005; 129:219-20.

3. Gammie JS, Banks MC, Fuhrman CR, Pham SM Griffith BP, Keenan RJ, et al. The pigtail catheter for pleural drainage: a less invasive alternative to tube thoracostomy. JSLS. 1999;3:57-61.

4. Waydhas C, Sauerland S. Pre-hospital pleural decompression and chest tube placement after blunt trauma: a systematic review. Resuscitation. 2006; 72:11-25C.

doi:10.1016/j.jtcvs.2009.10.067

\section{HYPOXEMIC REPERFUSION OF ISCHEMIC STATES PREVENTS MYOCARDIAL INJURY, ATTENUATING THE OXIDATIVE AND INFLAMMATORY RESPONSE} To the Editor:

We read with great interest the recent article by Abdel-Rahman and colleagues ${ }^{1}$ on the favorable effect of "hypoxic reoxygenation" in the attenuation of myocardial ischemia-reperfusion injury after cardioplegic arrest. The importance of progressive oxygen re-entry into ischemic tissues has been shown since 2001 in the brains of a porcine model of cardiac arrest. ${ }^{2,3}$ The favorable effect of the strategy was documented through better overall neurological performance, less lipid peroxidation, ${ }^{2}$ and attenuation of the brain morphologic changes. ${ }^{3}$ Because of malondialdehyde (MDA) increase, oxidative stress has been theoretically implicated, thought not confirmed by the measurement of reactive oxygen species (ROS).

With regard to the current study, 3 comments are to be addressed. First, the authors deduced oxidative myocardial injury by determining MDA concentrations in coronary sinus blood without comparative reference MDA concentrations at the distal site. Nevertheless, ROS seem to be directly cardiotoxic, independent of the site of their 